\title{
UNIFORM ASYMPTOTIC APPROXIMATION OF MATHIEU FUNCTIONS
}

\author{
T. M. Dunster
}

ABSTRACT. Uniform asymptotic approximations are derived for solutions of Mathieu's equation

$$
\frac{d^{2} w}{d z^{2}}=\{2 q \cos (2 z)-a\} w
$$

for $a$ and $q$ real, and $z$ complex. These are uniformly valid for $q$ large and $a$ lying in the interval $-2 q \leq a \leq(2-d) q, \quad(d>0)$, for all real or complex values of $z$. The approximations involve both elementary functions (LiouvilleGreen) and Whittaker functions. These results are derived by an application of a recent asymptotic theory of a coalescing turning point and simple pole in the complex plane. The new asymptotic approximations are then analytically continued around infinity, to derive a uniform asymptotic approximation between the characteristic exponent $v$ and the parameters $a$ and $q$. Error bounds are either included or available for all approximations.

\section{Introduction}

In this paper we shall derive uniform asymptotic approximations for solutions of Mathieu's equation

$$
\frac{d^{2} w}{d z^{2}}=\{2 q \cos (2 z)-a\} w
$$

for $z$ complex, and $q$ and $a$ real with $q \rightarrow \infty$. The approximations will be uniformly valid for

$$
-2 q \leq a \leq(2-d) q
$$

( $d$ an arbitrary small positive constant), with $z$ lying in the semi-infinite strip $0 \leq$ $\Re(z) \leq \pi, \Im(z) \geq 0$. Moreover, explicit error bounds for the difference between the approximations and exact solutions will be available. Extensions of our results to all other values of $z$ can be achieved using various connection formulae that we give in Sections 2 and 5 below.

We note here that the semi-infinite strip we are considering includes the real interval $[0, \pi]$, and the semi-infinite lines $\Re(z)=0$ and $\Re(z)=\pi / 2$. Thus, asymptotic approximations can be recovered from our results for solutions of the following real

Received June 21, 1993, revised November 29, 1993.

1991 Mathematics Subject Classification: 33E10, 41A60, 34B30, 34E20.

Key words and phrases: Mathieu functions, asymptotic approximations, Mathieu's equation, turning point theory.

This research was supported by the National Science Foundation under grant DMS-9102834. 
forms of Mathieu's equation:

$$
\begin{array}{rlrl}
\text { Ordinary: } & \frac{d^{2} w}{d x^{2}}=\{2 q \cos (2 x)-a\} w, & & (0 \leq x \leq \pi), \\
\text { Modified (oscillatory case): } & \frac{d^{2} w}{d y^{2}}=\{-2 q \cosh (2 y)+a\} w, & (0 \leq y<\infty), \\
\text { Modified (exponential case): } & \frac{d^{2} w}{d y^{2}}=\{2 q \cosh (2 y)+a\} w, & (0 \leq y<\infty) .
\end{array}
$$

The most comprehensive study so far on the asymptotic behaviour of Mathieu functions has been undertaken by Barrett [2]. In this paper Barrett introduced numerically satisfactory solutions of Mathieu's equation ("base functions"), and subsequently derived asymptotic approximations for these functions (with either $a$ or $q$ large) in terms of elementary, Airy, and Bessel functions. Although Barrett's results cover a large parameter regime and order-of-magnitude error estimates are derived, explicit error bounds are not obtained. In some cases the results are obtained in a heuristic manner; for example, approximations are given in a region in the complex plane containing two coalescing turning points, when a rigorous general asymptotic theory currently only exists in the real variable case [11].

There have been a number of other investigations into the asymptotic ( $q$ large) behavior of Mathieu functions (see for example [8], [9], and [12]), but it seems such treatments also have been essentially heuristic. Generally, the results in the literature are restricted in the range of the variables $z$ and $a$, and error bounds have not been derived in a satisfactory manner. Most of these results have been derived from a study of Mathieu's equation in its trigonometric form, (1.1).

In its trigonometric form Mathieu's equation has an irregular singularity at infinity, with no finite singularities. In asymptotic investigations, other points of importance are the so-called turning points, where locally solutions change in character from oscillatory to exponential. The turning points of (1.1) in the case $q \rightarrow \infty$ are located at, or near, the zeroes of the coefficient of the non-derivative term, that is, where

$$
2 q \cos (2 z)-a=0
$$

Denoting these turning points by $z_{t}$ we have

$$
z_{t}=\frac{1}{2} \arccos \left(\frac{a}{2 q}\right)
$$

which shows that they are infinite in number, and in the real parameter case are located on the real $z$-axis when $-2 q \leq a \leq 2 q$. Furthermore, if $a$ is fixed and $q \rightarrow \infty$, the turning points are then located close to the points $z= \pm \pi / 4, \pm 3 \pi / 4, \pm 5 \pi / 4, \ldots$. In the strip $0 \leq \Re(z) \leq \pi, \Im(z) \geq 0$, there are two turning points for our parameter range. Note that they are bounded away from one another if $a$ remains bounded as $q \rightarrow \infty$; however, for the range (1.2) we are considering, the turning points do not remain bounded away from each other, as we shall see below.

It is appropriate at this point to record some well-known basic properties of Mathieu's equation (1.1) and its solutions, which are relevant to this paper. For further details the reader is referred to an excellent introduction by F. M. Arscott [1, Chapters 2-6], and the monograph by J. Meixner and F. W. Schäfke [7]. 
Equation (1.1) is a periodic equation, since it remains unchanged if $z$ is replaced by $z+\pi$. Also, the equation is invariant under $z \rightarrow-z$, and consequently if $w(z)$ is any solution, so are the functions $w(n \pi \pm z)$ for any integer $n$.

There always exists one or more pseudo-periodic (or Floquet) solutions $w(z)$ having the property that $w(z+\pi)=M^{ \pm 1} w(z)$, for some number $M$ that depends on the parameters, but not on the independent variable $z$. We shall use the standard notation of

$$
M=e^{v \pi i},
$$

where $v$ is the so called characteristic exponent.

Of particular interest in many physical and mathematical problems is the case $M=$ 1 or -1 , so that there exists at least one basically-periodic solution, that is, a solution having period ${ }^{1} \pi$ or $2 \pi$. For each prescribed value of $q$ there exist a countably infinite number of values for $a$ for which $M= \pm 1$. Although there are various expressions for these characteristic values of $a$, no one expression is suitable for all ranges (large or small) of $q$. Moreover, these expressions are somewhat complicated (except in the case $q=0$ ), involving for example a combination of continued fractions and recursion relations.

When $q \rightarrow \infty$ the characteristic values of $a$ can be approximated by

$$
a=-2 q+2(2 m+1) q^{1 / 2}-\frac{(2 m+1)^{2}+1}{8}+O\left(m^{3} q^{-1 / 2}\right) \quad(m=0,1,2, \ldots),
$$

and for each of these values the basically-periodic solution is either even or odd (often denoted by $c e_{m}(q, z)$ or $\left.s e_{m+1}(q, z)\right)$. Thus, in the important case when a basicallyperiodic solution exists, we see from (1.6) that each characteristic value of $a$ becomes unbounded as $q \rightarrow \infty$.

Consequently, the turning points are not necessarily bounded away from one another and can coalesce. In fact, from (1.4) and (1.6) we notice that the two turning points in the strip $0 \leq \Re(z) \leq \pi, \Im(z) \geq 0$ satisfy

$$
z_{t}=\pi / 2 \pm o(1)
$$

when $q \rightarrow \infty$ and $a$ takes a characteristic value corresponding to (1.6) for each $m$ satisfying

$$
0 \leq m \leq o\left(q^{1 / 2}\right)
$$

Comparing (1.2) and (1.6), we see that our approximations will certainly include, but not be restricted to, the important characteristic values of $a$ which satisfy (1.6) and (1.8). We achieve this by transforming Mathieu's equation (1.1) into an algebraic form which, instead of having two coalescing turning points, has a coalescing turning point and a simple pole (see equation (3.3) below). We then apply a recent asymptotic theory which has been developed for this class of problem [3], which yields uniform asymptotic solutions in terms of Whittaker's confluent hypergeometric functions.

Although a real-variable asymptotic theory is available for the problem of two coalescing turning points [10], and hence in principle could be applicable to the present

\footnotetext{
${ }^{1}$ There is at most one independent basically-periodic solution, except in the trivial case $q=0$.
} 
problem, we use instead the complex-variable theory of [3]. There are several significant advantages of working in the complex domain in the present case. Although the most obvious one is that the subsequent results will be more general, perhaps more importantly one can achieve an identification of standard solutions of (1.1) with the asymptotic solutions, which would be difficult or impossible in the real-variable case.

Specifically, we shall at first focus our attention on four particular solutions of Mathieu's equation in its algebraic form (3.3), one of which is recessive at the simple pole at the origin, the other three being recessive in certain sectors at infinity in the complex plane. Since recessiveness at a singularity defines a solution uniquely, we will be able to match the asymptotic approximations derived from [3] with these four solutions. These approximations will hold uniformly in certain subdomains of the complex plane, and will be valid for $q \rightarrow \infty$ with (1.2) holding. Moreover, the four solutions will comprise a numerically satisfactory set in the complex domain we are considering.

Although only two of the four recessive solutions can be regarded as standard Mathieu functions, we will be able to construct uniform asymptotic approximations for all standard Mathieu functions, such as the Floquet solutions on the real interval $0 \leq z \leq \pi$, via certain connection formulae. Also, as a by-product of the derivation of one of these connection formulae, we shall obtain an asymptotic relationship between the parameters $q, a$, and the characteristic exponent $v$, again uniformly valid for $q \rightarrow \infty$ with (1.2) holding. This relationship will then give, as a special case, estimates for the characteristic values of $a$, as in (1.6) above, but considerably more powerful since the new estimates will be uniformly valid for the large range (1.2), and explicit bounds will be available for all error terms. This new relationship is more general than previous asymptotic results for the eigenvalues of Mathieu's equation (see, for example [5] and [6]).

\section{Mathieu functions: Definitions, fundamental properties, connection formulae}

In the notation of Meixner and Schäfke [7] a Floquet solution can be defined by

$$
m e_{v}(z, q)=e^{i v z} \sum_{r=-\infty}^{\infty} c_{v, 2 r} e^{2 r i z}
$$

where the coefficients $c_{v, 2 r}$ satisfy

$$
\begin{gathered}
q c_{v, 2 r-2}+\left\{(2 r+v)^{2}-a\right\} c_{v, 2 r}+q c_{v, 2 r+2}=0, \\
\lim _{r \rightarrow \pm \infty} c_{v, 2 r}=0
\end{gathered}
$$

subject to the normalizing condition

$$
\sum_{r=-\infty}^{\infty}\left(c_{v, 2 r}\right)^{2}=1
$$

We remark that $(2.2 \mathrm{~b})$ does not automatically hold for arbitrary values of the parameters $q, a$, and $v$, but rather only for a specific value or values of one parameter when the other two are prescribed.

The importance of $m e_{v}(z, q)$ derives from its pseudo-periodicity property

$$
m e_{v}(z+\pi, q)=e^{v \pi i} m e_{v}(z, q),
$$


which holds for any real or complex value of $z$. An accompanying solution is given by

$$
m e_{-v}(z, q)=m e_{v}(-z, q)
$$

and since

$$
m e_{-v}(z+\pi, q)=e^{-v \pi i} m e_{-v}(z, q),
$$

it too is pseudo-periodic. From (2.1) and (2.5) we note in passing a relation that we shall use later

$$
m e_{-v}(0, q)=m e_{v}(0, q)=\sum_{r=-\infty}^{\infty} c_{v, 2 r}
$$

Some authors use the pseudo-periodic functions (2.1) and (2.5) as a fundamental pair of solutions of Mathieu's equation (in the real-variable case), whilst some others choose

$$
c e_{v}(z, q)=\frac{1}{2}\left[m e_{v}(z, q)+m e_{-v}(z, q)\right], \quad s e_{v}(z, q)=\frac{1}{2 i}\left[m e_{v}(z, q)-m e_{-v}(z, q)\right],
$$

which are, respectively, even and odd pseudo-periodic solutions. However, it is important to note that neither the former nor latter pairs remain linearly independent for all parameter ranges of $q$ and $v$. In particular, when $v$ is an integer (and these solutions are basically-periodic), $m e_{v}(z, q)$ and $m e_{-v}(z, q)$ are multiples of one another, and one of the odd/even pair vanishes identically.

As mentioned in $\S 1$, we shall construct uniform asymptotic approximations for four Mathieu functions which form a numerically satisfactory set in the complex plane. The first of these we denote by $m_{v}^{(0)}(z, q)$, and define by

$$
m_{v}^{(0)}(z, q)=i e^{v \pi i / 2}\left[m e_{v}^{\prime}(\pi / 2, q)\right]^{-1} \sum_{r=-\infty}^{\infty}(-1)^{r} c_{v, 2 r} \sin \{(2 r+v)(z-\pi / 2)\},
$$

where the coefficients $c_{v, 2 r}$ are the same as those given above. The fundamental property of this function is that it is odd about the point $z=\pi / 2$ :

$$
m_{v}^{(0)}(\pi / 2+z, q)=-m_{v}^{(0)}(\pi / 2-z, q) .
$$

The existence of a Mathieu function having the property (2.10) is well-known (e.g., see [1, p. 27]), but it does not seem to appear in the literature as a standard solution. As noted in $\S 1$, its importance for our application is its recessiveness at a certain point in the complex plane. In particular

$$
m_{v}^{(0)}(z, q)=(z-\pi / 2)+O\left\{(z-\pi / 2)^{3}\right\} \quad(z \rightarrow \pi / 2),
$$

which follows from (2.9) and the identity

$$
m_{v}^{(0)^{\prime}}(\pi / 2, q)=i e^{v \pi i / 2}\left[m e_{v}^{\prime}(\pi / 2, q)\right]^{-1} \sum_{r=-\infty}^{\infty}(2 r+v)(-1)^{r} c_{v, 2 r}=1 .
$$

The choice of normalizing constant in (2.9) ensures that $m_{v}^{(0)}(z, q)$ exists and is not identically zero for all values of the parameters, as can be seen from (2.12). Since $z=$ $\pi / 2$ is an ordinary point of equation (1.1), it is evident that all solutions independent 
of $m_{v}^{(0)}(z, q)$ are non-vanishing as $z \rightarrow \pi / 2$. Therefore, the property (2.11) is a unique characteristic of the solution $m_{v}^{(0)}(z, q)$.

A relationship between $m_{v}^{(0)}(z, q)$ and the Floquet solutions is given by the following proportionality equation

$$
m_{v}^{(0)}(z, q)=k_{v}^{(0)}(q)\left[e^{-v \pi i / 2} m e_{v}(z, q)-e^{v \pi i / 2} m e_{-v}(z, q)\right]
$$

which follows as a consequence of (2.4), (2.6), and (2.10). An expression for the constant of proportionality can be obtained by setting $z=0$ in the equation, yielding

$$
k_{v}^{(0)}(q)=\frac{i m_{v}^{(0)}(0, q)}{2 \sin \{v \pi / 2\} m e_{v}(0, q)}=\frac{e^{v \pi i / 2}}{2 m e_{v}^{\prime}(\pi / 2, q)} .
$$

Our other three fundamental solutions are characterized by their behaviour at infinity in the complex plane. To investigate these, it is convenient now to convert (1.1) into an algebraic form via the change of independent variable

$$
\zeta=-\cos (z) .
$$

The minus sign is introduced merely for convenience, so that the strip $0 \leq \Re(z) \leq \pi$, $\Im(z) \geq 0$ is mapped to the upper half-plane $\Im(\zeta) \geq 0$. With this new independent variable, Mathieu's equation takes the form

$$
\frac{d^{2} w}{d \zeta^{2}}+\frac{\zeta}{\left(\zeta^{2}-1\right)} \frac{d w}{d \zeta}+\left(\frac{4 q \zeta^{2}-2 q-a}{\zeta^{2}-1}\right) w=0 .
$$

The particular choice of the range of $\arg (\zeta)$ is not critical, as long as it is used consistently; here and throughout we specify that

$$
0 \leq \arg (\zeta) \leq \pi
$$

Expansions for solutions of (2.16) have been obtained in terms of Bessel functions, viz.

$$
\sum_{r=-\infty}^{\infty}(-1)^{r} c_{v, 2 r} \mathcal{C}_{v+2 r}(2 \sqrt{q} \zeta)
$$

where $\mathcal{C}_{v}(\zeta)$ denotes a Bessel function of order $v$ and complex argument $\zeta$, and again the coefficients $c_{v, 2 r}$ are those given by $(2.2 \mathrm{a}, \mathrm{b})$; (see [1, pp. 89-91]). The series (2.18) converge absolutely and uniformly for $|\zeta|>1$, except in the special case when $\mathcal{C}=J$ and $v$ is an integer, when the series converges for all $\zeta$. Thus, the Bessel function series (2.18) have usually been of interest only in the study of modified Mathieu functions, since in general they do not converge on the real $z$-axis. However, we shall derive asymptotic approximations for these solutions which are valid in domains containing part of the real $z$-axis, thus in effect giving their analytic continuation.

Two solutions which we are particularly interested in involve Hankel functions, which we denote by

$$
m_{v}^{(3,4)}(z, q)=\left[m e_{v}(0, q)\right]^{-1} \sum_{r=-\infty}^{\infty}(-1)^{r} c_{v, 2 r} H_{v+2 r}^{(1,2)}(2 \sqrt{q} \zeta)
$$

This notation is in accord with Meixner and Schäfke [7], who denote solutions of the (real) modified Mathieu equation having similar Hankel function expansions by $M_{v}^{(3,4)}(y, q)$. 
The characterizing behaviour of the functions defined by (2.19) is

$$
m_{v}^{(3,4)}(z, q) \sim H_{v}^{(1,2)}(2 \sqrt{q} \zeta)
$$

as $\zeta \rightarrow \infty$, that is, as $\Im(z) \rightarrow \infty$. Thus, from well-known properties of Hankel functions of large argument [10, p. 238], the following behaviour as $\zeta \rightarrow \infty$ should be noted

$$
m_{v}^{(3)}(z, q) \sim\left(\frac{1}{\pi \sqrt{q} \zeta}\right)^{1 / 2} e^{2 i \sqrt{q} \zeta-v \pi i / 2-\pi i / 4} \quad(-\pi+\delta \leq \arg (\zeta) \leq 2 \pi-\delta),
$$

and

$$
m_{v}^{(4)}(z, q) \sim\left(\frac{1}{\pi \sqrt{q} \zeta}\right)^{1 / 2} e^{-2 i \sqrt{q} \zeta+v \pi i / 2+\pi i / 4} \quad(-2 \pi+\delta \leq \arg (\zeta) \leq \pi-\delta) .
$$

Therefore in particular $m_{v}^{(3)}(z, q)$ is recessive (i.e., exponentially small) as $\zeta \rightarrow \infty e^{\pi i / 2}$ $(z \rightarrow \pi / 2+i \infty)$, and $m_{v}^{(4)}(z, q)$ is recessive as $\zeta \rightarrow \infty e^{-\pi i / 2}(z \rightarrow 3 \pi / 2+i \infty)$. Also, both functions can be characterized by their purely oscillatory behaviour as $z \rightarrow \pi+i \infty$, and as such represent incoming and outgoing waves in scattering problems which involve the oscillatory modified Mathieu equation.

It should be emphasized that the series (2.19) converge for values of $\zeta$ which correspond to $z$ lying in the upper half-plane $\Im(z)>0$ (with a certain neighbourhood of the real axis excluded), and as such no information about $m_{v}^{(3)}(z, q)$ or $m_{v}^{(4)}(z, q)$ in the lower half-plane $\Im(z)<0$ can be inferred from these representations.

The fourth of our fundamental set of Mathieu functions is taken to be the function $m_{v}^{(3)}(z+\pi, q)$, whose representation is the same as $(2.19)$ for $m_{v}^{(3)}(z, q)$, except with $\zeta$ replaced by $\zeta e^{-\pi i}$. This solution then has the asymptotic behaviour

$$
m_{v}^{(3)}(z+\pi, q) \sim H_{v}^{(1)}\left(2 \sqrt{q} \zeta e^{-\pi i}\right)
$$

as $\zeta \rightarrow \infty$, and hence is recessive as $\zeta \rightarrow \infty e^{3 \pi i / 2}(z \rightarrow-\pi / 2+i \infty)$.

The large parameter asymptotic approximations we shall derive for $m_{v}^{(4)}(z, q)$ and $m_{v}^{(3)}(z+\pi, q)$, as well as that for $m_{v}^{(0)}(z, q)$, will hold in certain subdomains of the half-plane $0 \leq \arg (\zeta) \leq \pi$ and the union of these subdomains of asymptotic validity will cover this half-plane. Although only $m_{v}^{(3)}(z, q)$ is recessive at infinity in the halfplane, in any given region, one of $m_{v}^{(4)}(z, q), m_{v}^{(3)}(z+\pi, q)$ or $m_{v}^{(0)}(z, q)$ will serve as a numerically satisfactory companion to $m_{v}^{(3)}(z, q)$.

Further properties of $m_{v}^{(3)}(z, q)$ and $m_{v}^{(4)}(z, q)$ that we require can be obtained directly from (2.19) and well-known properties of Hankel functions (see [10, p. 239]). Specifically, for all $z$,

$$
\begin{gathered}
m_{-v}^{(3,4)}(z, q)=e^{ \pm v \pi i} m_{v}^{(3,4)}(z, q), \\
m_{v}^{(3)}(z+\pi, q)=2 \cos (v \pi) m_{v}^{(3)}(z, q)+e^{-v \pi i} m_{v}^{(4)}(z, q), \\
m_{v}^{(4)}(z+\pi, q)=-e^{v \pi i} m_{v}^{(3)}(z, q) .
\end{gathered}
$$


Note that (2.25) and (2.26) allow the extension of the asymptotic results of this paper to all $z$ in $\Im(z) \geq 0$.

Next we consider the problem of determining the connection of $m_{v}^{(3)}(z, q)$ and $m_{v}^{(4)}(z, q)$ with the Floquet solutions. To achieve this we first observe that there exists a constant $k_{v}(q)$ such that

$$
m e_{-v}(z, q)=2 k_{v}(q) \sum_{r=-\infty}^{\infty}(-1)^{r} c_{v, 2 r} J_{v+2 r}(2 \sqrt{q} \zeta)
$$

a result which follows from the shared property (2.6) of both sides of the equation. The constant $k_{v}(q)$ cannot easily be determined exactly, since the Bessel function series does not generally converge on the real $z$-axis and $m e_{-v}(z, q)$ does not have a known behaviour as $\Im(z) \rightarrow \infty$. We shall, however, derive a uniform asymptotic approximation for this constant (for the case $q$ large, which we are considering). We note in passing that when $v=n$ is an integer, the Bessel function series converges on the real axis, and in this case we can set $z=\pi / 2(\zeta=0)$ in (2.27) to obtain

$$
k_{n}(q)=\frac{m e_{-n}(\pi / 2, q)}{2 c_{n, 0}} .
$$

The relationship between $m e_{-v}(z, q)$ and $m_{v}^{(3)}(z, q)$ and $m_{v}^{(4)}(z, q)$ can be obtained from (2.19), (2.27), and the relation $2 J_{v}(z)=H_{v}^{(1)}(z)+H_{v}^{(2)}(z)$. Thus

$$
m e_{-v}(z, q)=m e_{v}(0, q) k_{v}(q)\left[m_{v}^{(3)}(z, q)+m_{v}^{(4)}(z, q)\right] .
$$

Hence, from replacing $v$ by $-v$ in (2.29), and using (2.7) and (2.24), we have

$$
m e_{v}(z, q)=m e_{v}(0, q) k_{-v}(q)\left[e^{v \pi i} m_{v}^{(3)}(z, q)+e^{-v \pi i} m_{v}^{(4)}(z, q)\right] .
$$

On setting $z=0$ in (2.29), and using (2.25) with $z=0$, it is straightforward to show that

$$
k_{v}(q)=e^{-v \pi i}\left[m_{v}^{(3)}(\pi, q)-e^{v \pi i} m_{v}^{(3)}(0, q)\right]^{-1},
$$

which we shall use to obtain a uniform asymptotic approximation for $k_{v}(q)$ (see equation (5.23) below).

Next, from (2.4)-(2.6) we observe that

$$
m e_{ \pm v}(\pi / 2+z, q)=e^{ \pm v \pi i} m e_{\mp v}(\pi / 2-z, q) .
$$

Hence, with the aid of (2.29) and (2.30), we derive the connection formulae

$$
\begin{aligned}
& m_{v}^{(3)}(\pi / 2+z, q)=\frac{\sin \left\{\left(v-\omega_{v}(q)\right) \pi\right\}}{\sin (v \pi)} m_{v}^{(3)}(\pi / 2-z, q) \\
& +e^{-v \pi i} \frac{\sin \left\{\left(2 v-\omega_{v}(q)\right) \pi\right\}}{\sin (v \pi)} m_{v}^{(4)}(\pi / 2-z, q), \\
& m_{v}^{(4)}(\pi / 2+z, q)=e^{v \pi i} \frac{\sin \left(\omega_{v}(q) \pi\right)}{\sin (v \pi)} m_{v}^{(3)}(\pi / 2-z, q) \\
& -\frac{\sin \left\{\left(v-\omega_{v}(q)\right) \pi\right\}}{\sin (v \pi)} m_{v}^{(4)}(\pi / 2-z, q),
\end{aligned}
$$


where, for convenience, we have introduced a parameter $\omega_{v}(q)$ by the relation

$$
\exp \left\{i \omega_{v}(q) \pi\right\}=\frac{k_{-v}(q)}{k_{v}(q)}=\frac{m_{v}^{(3)}(0, q)+m_{v}^{(4)}(0, q)}{e^{v \pi i} m_{v}^{(3)}(0, q)+e^{-v \pi i} m_{v}^{(4)}(0, q)} .
$$

The fractions (here and elsewhere) may be replaced by their limiting values in the event of both numerator and denominator vanishing for certain parameter values.

Since an (asymptotic) expression can be derived for $\omega_{v}(q)$ (via the approximation (5.23) for $k_{v}(q)$ ), equations (2.33) and (2.34) supply the analytic continuation of $m_{v}^{(3)}(z, q)$ and $m_{v}^{(4)}(z, q)$ into the lower half-plane. Hence, the connection formulae (2.25), (2.26), (2.33), and (2.34) enable the subsequent large parameter asymptotic results, which will be valid in the semi-infinite strip $0 \leq \Re(z) \leq \pi, \Im(z) \geq 0$, to be extended to all values of $z$ if desired.

The final connection formula that we require is the one of the form

$$
m_{v}^{(0)}(z, q)=A m_{v}^{(3)}(z, q)+B m_{v}^{(4)}(z, q)
$$

and asymptotic expressions for the constants $A$ and $B$ will be found via a matching of the corresponding uniform asymptotic approximations (see equation (5.18) below). We note that the following expressions can be obtained from (2.13) and (2.30)

$$
\begin{gathered}
A=\frac{e^{v \pi i / 2} k_{v}^{(0)}(q)\left\{k_{-v}(q)-k_{v}(q)\right\}}{m e_{v}(0, q)} \\
B=\frac{e^{v \pi i / 2} k_{v}^{(0)}(q)\left\{e^{-2 v \pi i} k_{-v}(q)-k_{v}(q)\right\}}{m e_{v}(0, q)}
\end{gathered}
$$

although we shall not employ these.

\section{Uniform asymptotic solutions involving Whittaker functions}

To apply the theory of [3], let us make the following preliminary Liouville transformation on Mathieu's equation (2.16)

$$
\begin{gathered}
t=\zeta^{2}=\cos ^{2}(z), \\
\tilde{w}(t)=\{\cos (z) \sin (z)\}^{1 / 2} w(z) .
\end{gathered}
$$

This yields the following algebraic form of Mathieu's equation, which will be the focus of our attention from now on,

$$
\frac{d^{2} \tilde{w}}{d t^{2}}=\left\{u^{2} f(\tilde{a}, t)+g(t)\right\} \tilde{w}
$$

where

$$
f(\tilde{a}, t)=\frac{t-\tilde{a}}{t(1-t)}, \quad g(t)=-\frac{4 t^{2}-4 t+3}{16 t^{2}(1-t)^{2}},
$$

and, for convenience, we have introduced the new parameters

$$
\tilde{a}=\frac{1}{4}\left(\frac{a}{q}+2\right), \quad u=\sqrt{q}
$$


We shall examine the asymptotic behaviour of solutions of (3.3) as $u \rightarrow \infty$, for $t$ lying in the plane having a cut along the positive real axis, such that

$$
0 \leq \arg (t) \leq 2 \pi
$$

(see Figures 1 and 2). We denote this region in the cut $t$-plane by $\tilde{\mathbf{D}}$. Equation (3.3) is characterized by $f(\tilde{a}, t)$ having simple poles at $t=0,1$ and a turning point at $t=\tilde{a}$.

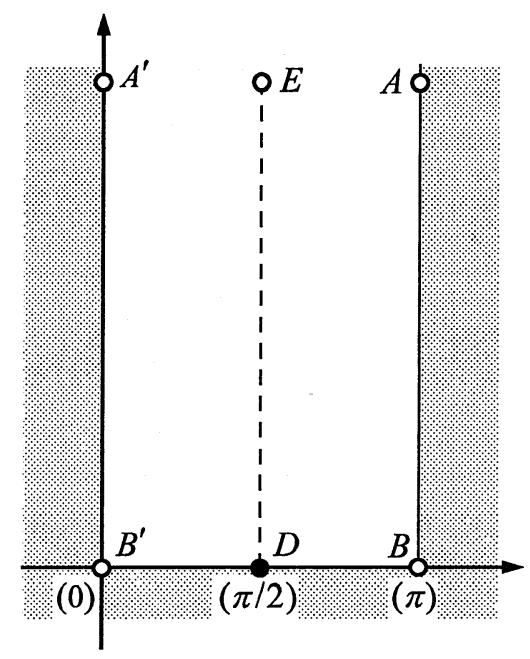

FiguRe 1. $z$-plane

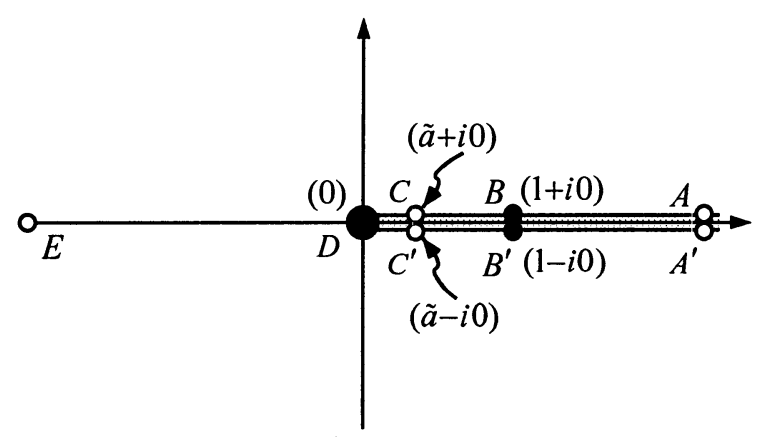

FiguRe 2. $t$-plane

The parameter range (1.2) is equivalent to

$$
0 \leq \tilde{a} \leq 1-d \quad(d>0)
$$

and hence the turning point is located on the cut along the positive real axis between the two poles, and coalesces with the pole at the origin in the critical case $\tilde{a}=0$.

The point ${ }^{2} t=\tilde{a}-i 0$ corresponds to a turning point in the $z$-plane at $z=$ $\arccos (\sqrt{\tilde{a}})$, which lies in the interval $(0, \pi / 2]$, and the point $t=\tilde{a}+i 0$ corresponds to a turning point in the $z$-plane at $z=\arccos (-\sqrt{\tilde{a}})$, which lies in the interval $[\pi / 2, \pi)$. These two turning points in the $z$-plane coalesce at $z=\pi / 2$ in the critical case $\tilde{a}=0$.

\footnotetext{
${ }^{2}$ Here and throughout, we denote points above or below a cut by $\pm i 0$.
} 
Note that $f(\tilde{a}, t)$ is positive in $(-\infty, 0) \cup(\tilde{a}, 1)$, and negative in $(0, \tilde{a}) \cup(1, \infty)$; in the former interval, solutions are exponential in behaviour as $u \rightarrow \infty$, and in the latter they are oscillatory in behaviour (regardless of the branch of $\arg (t)$ ).

Following [3, equation (4.6)], we introduce the non-negative parameter

$$
\alpha=\frac{2 \tilde{a}}{\pi} \int_{0}^{1}\left\{\frac{1-s}{s(1-\tilde{a} s)}\right\}^{1 / 2} d s
$$

which has the property that $\alpha \rightarrow 0$ as $\tilde{a} \rightarrow 0$. Note that $\alpha$ can be expressed in terms of the elliptic integral of the second kind:

$$
\alpha=\frac{4 \sqrt{\tilde{a}}}{\pi} E\left(\operatorname{sn}^{-1}(\sqrt{\tilde{a}}) \mid 1 / \tilde{a}\right)
$$

where

$$
E\left(\operatorname{sn}^{-1}(x) \mid m\right)=\int_{0}^{x}\left\{\frac{1-m t^{2}}{1-t^{2}}\right\}^{1 / 2} d t .
$$

Our next step is to make a Liouville transformation on (3.3) with new independent and dependent variables $\xi$ and $W(\xi)$; the transformed equation for $W(\xi)$ will keep the essential features of (3.3), with the "large" factor $u^{2}$ multiplying the function $(\xi-\alpha) / \xi$, which has a simple pole at $\xi=0$ and a zero at $\xi=\alpha$. To this end, from [3, equation (4.4)], we define $\xi$ by

$$
\int_{\alpha}^{\xi}\left\{\frac{\tau-\alpha}{\tau}\right\}^{1 / 2} d \tau=\int_{\tilde{a}}^{t}\left\{\frac{s-\tilde{a}}{s(1-s)}\right\}^{1 / 2} d s
$$

where the branches on both sides are chosen so that $\xi(t)$ is

(i) an analytic function of $t$ at $t=0$ and $t=\tilde{a}$,

(ii) negative when $t$ is negative,

(iii) a continuous function for $t$ lying in the plane having a branch cut along the positive real axis from $t=1$ to $t=\infty$.

Figure 3 depicts the $\xi$ region $\hat{\mathbf{D}}$ corresponding to the $t$ region $\tilde{\mathbf{D}}$. We specify that for $\xi \in \hat{\mathbf{D}}$

$$
0 \leq \arg (\xi) \leq 2 \pi
$$

The points $t=0, \tilde{a}$ are mapped to $\xi=0, \alpha$, respectively, and we denote the point corresponding to $t=1$ by $\xi=\xi_{1}$. The bounding curve $A B$ emanating at an angle $\pi / 2$ from $\xi_{1}+i 0$ is the map of the upper part of the cut from $t=1$ to $t=\infty(\arg (t)=0)$; for all points $\xi$ on this curve

$$
\Re \int_{\xi_{1}+i 0}^{\xi}\left\{\frac{\tau-\alpha}{\tau}\right\}^{1 / 2} d \tau=0
$$

The curve $A^{\prime} B^{\prime}$ is the conjugate of $A B$.

The curve $G H$ emanating at an angle $\pi / 2$ from $\alpha+1 /(2 u)+i 0$ is defined by

$$
\Re \int_{\alpha+1 /(2 u)+i 0}^{\xi}\left\{\frac{\tau-\alpha}{\tau}\right\}^{1 / 2} d \tau=0 \quad(\Im(\xi) \geq 0),
$$

and $G^{\prime} H^{\prime}$ is the conjugate curve. These two curves separate $\hat{\mathbf{D}}$ into three regions, which we denote by $\hat{\mathbf{D}}_{0}, \hat{\mathbf{D}}^{+}$, and $\hat{\mathbf{D}}^{-}$, as depicted in Figure 3. The three regions, 


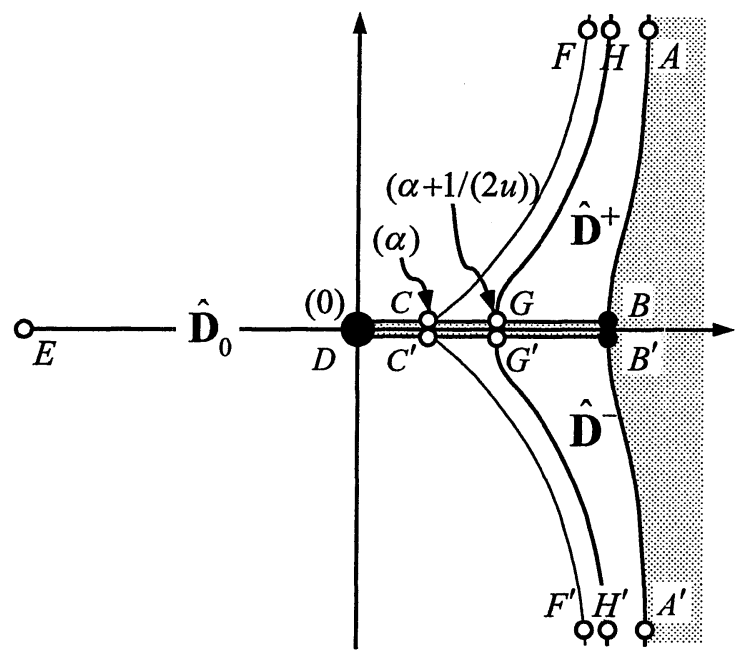

Figure 3. $\xi$-plane

which are defined to include all their boundary points, will be used below to describe the regions of asymptotic validity of certain solutions.

With the above new independent variable $\xi$ and a new dependent variable $W(\xi)$ defined by

$$
W(\xi)=\left\{\frac{\xi(t-\tilde{a})}{t(\xi-\alpha)(1-t)}\right\}^{1 / 4} \tilde{w}(t)
$$

we arrive at the transformed equation

$$
\frac{d^{2} W}{d \xi^{2}}=\left\{u^{2}\left(\frac{\xi-\alpha}{\xi}\right)-\frac{3}{16 \xi^{2}}+\frac{\hat{\psi}(\alpha, \xi)}{\xi}\right\} W .
$$

The branches of the factor multiplying $\tilde{w}(t)$ in (3.15) are to be taken so that this term is an analytic function of $\xi$ in $\hat{\mathbf{D}}$ (except at $\xi=\xi_{1}$ ), and is positive on the interval $-\infty<\xi<\xi_{1}$. In the notation of [3], we have $m=1 / 4$, and, from [3, equation (4.3)], we find that

$$
\hat{\psi}(\alpha, \xi)=\frac{3 \xi+2 \alpha}{16(\xi-\alpha)^{2}}+\frac{(\xi-\alpha)\left(t^{2}+4 \tilde{a} t-3 t-2 \tilde{a}\right)}{16(\tilde{a}-t)^{3}}
$$

which is analytic at all points in $\hat{\mathbf{D}}$, in particular at $\xi=0, \alpha$, and $\xi_{1}$.

Explicit integration of the left-hand side of (3.11) yields the relation

$$
\xi^{1 / 2}(\xi-\alpha)^{1 / 2}-\frac{\alpha}{2} \ln \left(\frac{2 \xi-\alpha+2 \xi^{1 / 2}(\xi-\alpha)^{1 / 2}}{\alpha}\right)=\int_{\tilde{a}}^{t}\left\{\frac{s-\tilde{a}}{s(1-s)}\right\}^{1 / 2} d s
$$

Hence we can show that $\xi \rightarrow \infty$ as $t \rightarrow \infty$ such that

$$
\xi-\frac{1}{2} \alpha \ln (4 \xi)-\frac{1}{2} \alpha+\frac{1}{2} \alpha \ln (\alpha)+O\left(\xi^{-1}\right)=2 i t^{1 / 2}+\alpha^{*}-\alpha \pi i / 2+O\left(t^{-1 / 2}\right)
$$


where

$$
\alpha^{*}=\int_{\tilde{a}}^{1}\left\{\frac{s-\tilde{a}}{s(1-s)}\right\}^{1 / 2} d s .
$$

This relationship will be of use later. In terms of the elliptic integral of the second kind (3.10), we have

$$
\alpha^{*}=2\{1-\tilde{a}\}^{1 / 2} E\left(\operatorname{sn}^{-1}\left(\{1-\tilde{a}\}^{1 / 2}\right) \mid 1 /\{1-\tilde{a}\}\right) .
$$

We shall also require information on the behaviour of $\xi$ as $t \rightarrow 0$, and this is readily found from (3.8) and (3.18) to be given by

$$
\xi=\frac{\tilde{a}}{\alpha} t+\frac{\tilde{a}^{2}-\alpha^{2}+\tilde{a} \alpha^{2}}{3 \alpha^{3}} t^{2}+O\left(t^{3}\right) .
$$

In the case when $\tilde{a}=\alpha=0$ the limiting form of (3.22) is applicable.

We now apply Theorem II of [3] to obtain three asymptotic solutions of (3.16) of the form

$$
\hat{W}^{(j)}(u, \alpha, \xi)=\hat{\mathcal{U}}_{u \alpha / 2,1 / 4}^{(j)}(2 u \xi)+\hat{\varepsilon}^{(j)}(u, \alpha, \xi) \quad(j=0,1,4),
$$

where $\mathcal{U}_{u \alpha / 2,1 / 4}^{(j)}(2 u \xi)$ denote the following Whittaker confluent hypergeometric functions (see [10, p. 260])

$$
\begin{gathered}
\hat{\mathcal{U}}_{u \alpha / 2,1 / 4}^{(0)}(2 u \xi)=\frac{\Gamma(u \alpha / 2+3 / 4)}{\gamma(u \alpha / 2) \Gamma(3 / 2)} M_{u \alpha / 2,1 / 4}(2 u \xi), \\
\hat{\mathcal{U}}_{u \alpha / 2,1 / 4}^{(1)}(2 u \xi)=e^{-\pi i / 4} \gamma(u \alpha / 2) W_{-u \alpha / 2,1 / 4}\left(2 u \xi e^{-\pi i}\right), \\
\hat{\mathcal{U}}_{u \alpha / 2,1 / 4}^{(4)}(2 u \xi)=\frac{e^{(2 u \alpha-3) \pi i / 4}}{\gamma(u \alpha / 2)} W_{u \alpha / 2,1 / 4}(2 u \xi),
\end{gathered}
$$

with

$$
\gamma(k)=k^{k} e^{-k} .
$$

Explicit bounds for the error terms $\hat{\varepsilon}^{(j)}(u, \alpha, \xi)$ and their derivatives are supplied by (4.80) of [3]. The paths of variation in these bounds, for the present case, are taken to connect $\xi$ with a reference point $\hat{\xi}^{(j)}$ prescribed as follows: for $j=0$ the reference point is taken to be $\hat{\xi}^{(0)}=0$, for $j=1$ we take $\hat{\xi}^{(1)}=\infty e^{\pi i}$, and for $j=4$ we take $\hat{\xi}^{(4)}$ to be the point at infinity lying on the curve $A B$ (corresponding to $t=\infty+i 0$, $z=\pi+i \infty)$.

Although the analysis of $[3, \S 4]$ is described for the principal range $-\pi \leq \arg (\xi) \leq \pi$, extension of the results to the present range of $0 \leq \arg (\xi) \leq 2 \pi$ is straightforward. We meet the essential requirement that the $t-\xi$ transformation be regular at the critical points $\xi=0, \alpha \pm i 0$. The error bounds (4.80) are valid in domains $\hat{\Delta}_{u \alpha / 2,1 / 4}^{(j)}$ ( $j=0,1,4)$ which meet the conditions [3, (i)-(iv) preceding equation (4.66)], with $\hat{S}_{\alpha}^{(1)}$ of that reference replaced by the larger region $\hat{\mathbf{D}}_{0}$, and $\hat{S}_{\alpha}^{(3)}$ replaced by the subdomain $\hat{\mathbf{D}}^{+}$.

With this extension to $0 \leq \arg (\xi) \leq 2 \pi$, we see that the bound for $\hat{\varepsilon}^{(1)}(u, \alpha, \xi)$ holds uniformly for all $\xi \in \hat{\mathbf{D}}$, and the bound for $\hat{\varepsilon}^{(4)}(u, \alpha, \xi)$ holds uniformly for $\xi \in \hat{\mathbf{D}}^{+}$. Note that in the latter case the region of validity $\hat{\mathbf{D}}^{+}$includes all points on the boundary $G H$. 
The bound for $\hat{\varepsilon}^{(0)}(u, \alpha, \xi)$ holds uniformly for all $\xi \in \hat{\mathbf{D}}$, provided $\frac{1}{2} u \alpha-\frac{3}{4} \notin \mathbf{N}$. If $\frac{1}{2} u \alpha-\frac{3}{4} \in \mathbf{N}$, then the bound is uniformly valid for $\xi \in \hat{\mathbf{D}}_{0}$. The extension of the bound for $\hat{\varepsilon}^{(0)}(u, \alpha, \xi)$ into $\hat{\mathbf{D}}^{+} \cup \hat{\mathbf{D}}^{-}$when $\frac{1}{2} u \alpha-\frac{3}{4} \in \mathbf{N}$ is still feasible since the monotonicity condition on the path of variation is not violated. However, the bounds would not be meaningful since they would imply that the error term $\hat{\varepsilon}^{(0)}(u, \alpha, \xi)$ is asymptotically larger than the Whittaker function approximant $\hat{\mathcal{U}}_{u \alpha / 2,1 / 4}^{(0)}(2 u \xi)$ (which becomes recessive in $\hat{\mathbf{D}}^{+} \cup \hat{\mathbf{D}}^{-}$when $\left.\frac{1}{2} u \alpha-\frac{3}{4} \in \mathbf{N}\right)$.

It is worth dwelling on this point a moment longer, since this an interesting complication in the asymptotic investigation of Mathieu's equation, or more generally in the theory of a coalescing turning point and simple pole, and two coalescing turning points. The essential complication is that for certain parameter values a solution which is recessive at one singularity can become recessive at a second singularity. The Whittaker function approximants $\hat{\mathcal{U}}_{u \alpha / 2,1 / 4}^{(0)}(2 u \xi)$ and $\hat{\mathcal{U}}_{u \alpha / 2,1 / 4}^{(4)}(2 u \xi)$ exhibit a similar dual recessiveness, but not for exactly the same parameter values as the solution they approximate. Thus for example, when $\frac{1}{2} u \alpha-\frac{3}{4} \in \mathbf{N}$ the Whittaker function $\hat{\mathcal{U}}_{u \alpha / 2,1 / 4}^{(0)}(2 u \xi)$ is recessive in $\tilde{\mathbf{D}}^{+}$, whereas $\hat{W}^{(0)}(u, \alpha, \xi)$ is recessive in $\hat{\mathbf{D}}^{+}$when $\frac{1}{2} u \alpha-\frac{3}{4}$ is close to a nonnegative integer (as $u \rightarrow \infty$ ). For this reason it is best to avoid (for all values of $\left.\frac{1}{2} u \alpha-\frac{3}{4}\right)$ use of the solution $\hat{W}^{(0)}(u, \alpha, \xi)$ in $\hat{\mathbf{D}}^{+}$if possible, and rely on connection formulae for the exact solution instead.

In a similar vein, we could extend the region of asymptotic validity for $\hat{\mathcal{U}}_{u \alpha / 2,1 / 4}^{(4)}(2 u \xi)$ into certain subdomains of $\hat{\mathbf{D}}_{0}$ (depending on the values of $\frac{1}{2} u \alpha-\frac{3}{4}$ ). Again, this would be an unnecessary complication, and we can content ourselves with employing $\hat{\mathcal{U}}_{u \alpha / 2,1 / 4}^{(0)}(2 u \xi)$ and $\hat{\mathcal{U}}_{u \alpha / 2,1 / 4}^{(1)}(2 u \xi)$ as a numerically satisfactory fundamental pair of solutions in $\hat{\mathbf{D}}_{0}$, and $\hat{\mathcal{U}}_{u \alpha / 2,1 / 4}^{(4)}(2 u \xi)$ and $\hat{\mathcal{U}}_{u \alpha / 2,1 / 4}^{(1)}(2 u \xi)$ as a numerically satisfactory fundamental pair of solutions in $\hat{\mathbf{D}}^{+}$.

The domain $\hat{S}_{\alpha}^{(2)}$ of [3] is not included in our range $0 \leq \arg (\xi) \leq 2 \pi$. However, we have an additional region $\hat{\mathbf{D}}^{-}$in $3 \pi / 2<\arg (\xi) \leq 2 \pi$, in which a recessive asymptotic solution is required. This is given simply by the solution

$$
\hat{W}^{(4)}\left(u, \alpha, \xi e^{-2 \pi i}\right)=\frac{e^{(2 u \alpha-3) \pi i / 4}}{\gamma(u \alpha / 2)} W_{u \alpha / 2,1 / 4}\left(2 u \xi e^{-2 \pi i}\right)+\hat{\varepsilon}^{(4)}\left(u, \alpha, \xi e^{-2 \pi i}\right),
$$

where $\hat{\varepsilon}^{(4)}\left(u, \alpha, \xi e^{-2 \pi i}\right)$ can be bounded for $\xi \in \hat{\mathbf{D}}^{-}$by the corresponding bound [3, equation (4.80)] for $\hat{\varepsilon}^{(4)}(u, \alpha, \bar{\xi})\left(\bar{\xi} \in \hat{\mathbf{D}}^{+}\right)$.

The characteristic behaviour of each asymptotic solution is as follows:

$$
\begin{array}{rlrl}
\hat{W}^{(0)}(u, \alpha, \xi) & \sim \frac{\Gamma(u \alpha / 2+3 / 4)}{\gamma(u \alpha / 2) \Gamma(3 / 2)}(2 u \xi)^{3 / 4} & (\xi \rightarrow 0), \\
\hat{W}^{(1)}(u, \alpha, \xi) \sim \gamma(u \alpha / 2) e^{(2 u \alpha-1) \pi i / 4}(2 u \xi)^{-u \alpha / 2} e^{u \xi} & (\xi \rightarrow \infty), \\
\hat{W}^{(4)}(u, \alpha, \xi) & \sim \frac{e^{(2 u \alpha-3) \pi i / 4}}{\gamma(u \alpha / 2)}(2 u \xi)^{u \alpha / 2} e^{-u \xi} & \left(\xi \in \hat{\mathbf{D}}^{+}, \xi \rightarrow \infty\right), \\
\hat{W}^{(4)}\left(u, \alpha, \xi e^{-2 \pi i}\right) & \sim \frac{e^{-(2 u \alpha+3) \pi i / 4}}{\gamma(u \alpha / 2)}(2 u \xi)^{u \alpha / 2} e^{-u \xi} & \left(\xi \in \hat{\mathbf{D}}^{-}, \xi \rightarrow \infty\right)
\end{array}
$$


We shall also require knowledge of the behaviour of $\hat{\mathcal{U}}_{u \alpha / 2,1 / 4}^{(0)}(2 u \xi)$ as $\xi \rightarrow \infty$ in $\hat{\mathbf{D}}$. As $\xi \rightarrow \infty$ with $0 \leq \arg (\xi)<3 \pi / 2$

$$
\begin{array}{r}
\hat{\mathcal{U}}_{u \alpha / 2,1 / 4}^{(0)}(2 u \xi) \sim \frac{\Gamma(u \alpha / 2+3 / 4)}{\gamma(u \alpha / 2) \Gamma(3 / 4-u \alpha / 2)}(2 u \xi)^{-u \alpha / 2} e^{u \xi} \\
-\frac{e^{-(2 u \alpha+1) \pi i / 4}}{\gamma(u \alpha / 2)}(2 u \xi)^{u \alpha / 2} e^{-u \xi}
\end{array}
$$

and as $\xi \rightarrow \infty$ with $\pi / 2<\arg (\xi) \leq 2 \pi$

$$
\begin{gathered}
\hat{\mathcal{U}}_{u \alpha / 2,1 / 4}^{(0)}(2 u \xi) \sim-\frac{e^{(2 u \alpha+1) \pi i / 2} \Gamma(u \alpha / 2+3 / 4)}{\gamma(u \alpha / 2) \Gamma(3 / 4-u \alpha / 2)}(2 u \xi)^{-u \alpha / 2} e^{u \xi} \\
-\frac{e^{-(2 u \alpha+1) \pi i / 4}}{\gamma(u \alpha / 2)}(2 u \xi)^{u \alpha / 2} e^{-u \xi}
\end{gathered}
$$

\section{Asymptotic solutions involving elementary functions}

Although the asymptotic solutions involving Whittaker functions given in the preceding section are uniformly valid in domains which contain the coalescing turning point and simple pole, we shall also employ the Liouville-Green (L-G) solutions of complex argument. The advantage of these is that they are simpler since they only involve elementary functions; the disadvantage is that they are not valid in the neighbourhood of the turning point.

We shall apply Theorem 3.1 of [10, Chapter 10], taking $n=1$, and not consider deriving asymptotic expansions, since expansions cannot similarly be derived for the Whittaker function approximations. The appropriate Liouville transformation is given by

$$
\begin{aligned}
& \sigma=\int_{0}^{t}\left\{\frac{s-\tilde{a}}{s(1-s)}\right\}^{1 / 2} d s, \\
& L(\sigma)=\left(\frac{t-\tilde{a}}{t(1-t)}\right)^{1 / 4} \tilde{w}(t),
\end{aligned}
$$

which transforms (3.3) into the form

$$
\frac{d^{2} L}{d \sigma^{2}}=\left\{u^{2}+\phi(\sigma)\right\} L
$$

where

$$
\phi(\sigma)=\frac{t^{2}-3 t+4 \tilde{a} t-2 \tilde{a}}{16(t-\tilde{a})^{3}} .
$$

The branches for the integrand of the right-hand side of (4.1) are taken so that $\sigma(t)$ is real and negative when $t$ is real and negative, and is continuous elsewhere in $\tilde{\mathbf{D}}$. The $\sigma$-domain corresponding to $\tilde{\mathbf{D}}$, which we denote by $\Xi$, is depicted in Figure 4 . The points $t=0, \tilde{a} \pm i 0,1 \pm i 0$ are mapped to $\sigma=0, \pm \alpha \pi i / 2, \alpha^{*} \pm \alpha \pi i / 2$, respectively, (where $\alpha^{*}$ is defined by equation (3.20) above). Note that $\phi(\sigma)$ is analytic at $\sigma=0$ and $\sigma=\alpha^{*} \pm \alpha \pi i / 2$, but not at $\sigma= \pm \alpha \pi i / 2$.

Figure 4 shows subdomains $\Xi^{+}, \Xi^{-}$, and $\Xi_{0}$, which are defined as follows. $\Xi_{0}$ is the 


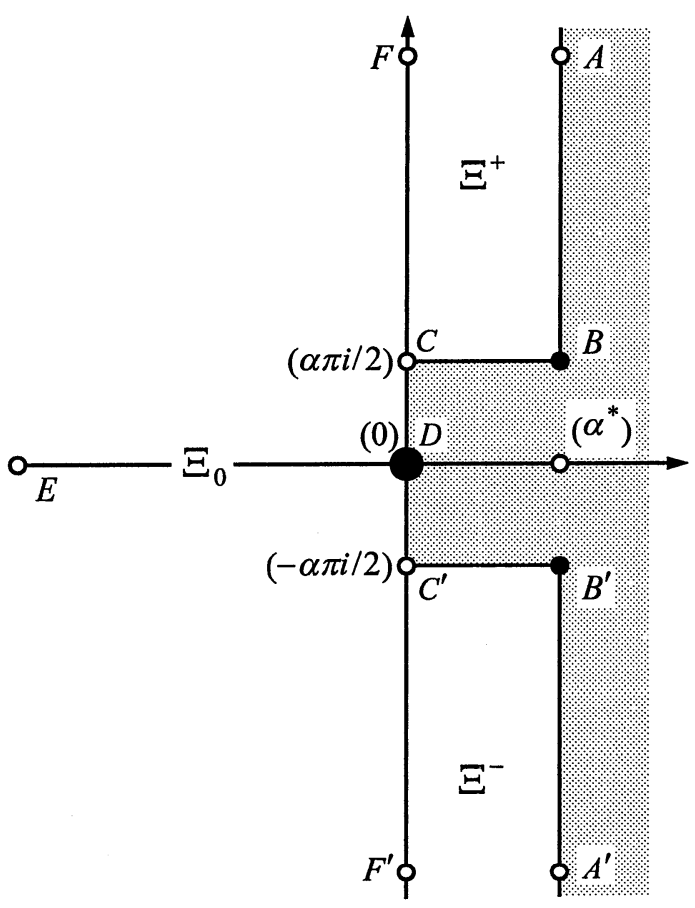

FiguRE 4. $\sigma$-plane

half-plane $\Re(\sigma)<0, \Xi^{+}$consists of all points in $\Xi$ satisfying $\Re(\sigma) \geq 0, \Im(\sigma) \geq \alpha \pi / 2$, and $\Xi^{-}$is the conjugate domain of $\Xi^{+}$. These definitions will be of use below.

To determine the regions of asymptotic validity for the solutions given by equations (3.02) and (3.03) of Theorem 3.1 of [10, Chapter 10], one must choose appropriate reference points. For the first of these solutions we choose the reference point to be at $\sigma=\infty e^{\pi i}$ (and denote the solution by $L_{1}(u, \sigma)$ ). For solutions of the form [10, Chapter 10, equation (3.03)] we choose two different reference points to yield two more asymptotic solutions; the first reference point is taken to be at $\sigma=\alpha^{*}+i \infty$ (denoting the solution by $L_{2}^{+}(u, \sigma)$ ), and the other we take to be at $\sigma=\alpha^{*}-i \infty$ (denoting the solution by $L_{2}^{-}(u, \sigma)$ ).

We therefore have the following L-G solutions of equation (4.3)

$$
\begin{gathered}
L_{1}(u, \sigma)=e^{u \sigma}\left\{1+\eta_{1}(u, \sigma)\right\}, \\
L_{2}^{ \pm}(u, \sigma)=e^{-u \sigma}\left\{1+\eta_{2}^{ \pm}(u, \sigma)\right\},
\end{gathered}
$$

with error bounds of the form

$$
|\eta(u, \sigma)|,\left|\frac{\partial \eta(u, \sigma)}{2 u \partial \sigma}\right| \leq \frac{1}{u} \int_{\infty}^{\sigma}|\phi(\tau) d \tau| \exp \left\{\frac{1}{u} \int_{\infty}^{\sigma}|\phi(\tau) d \tau|\right\} .
$$

The lower limits of integration in (4.7) are taken at the reference points at infinity described above; the paths of integration consist of a finite chain of $R_{2}$ arcs such that, as $\tau$ passes from the reference point at infinity to $\sigma, \Re(\tau)$ is nondecreasing for $\eta_{1}(u, \sigma)$, and $\Re(\tau)$, is nonincreasing for $\eta_{2}^{ \pm}(u, \sigma)$. The paths also must avoid neighbourhoods of the singularities $\sigma= \pm \alpha \pi i / 2$. 
The bounds (4.7) are uniformly valid in subdomains of $\Xi$ in which these "progressive path" requirements are met. Thus, $\eta_{1}(u, \sigma)$ is bounded by (4.7) for $\sigma$ in $\Xi$ with neighbourhoods of the points $\sigma= \pm \alpha \pi i / 2$ excluded, $\eta_{2}^{+}(u, \sigma)$ is bounded by (4.7) for $\sigma$ in $\Xi_{0} \cup \Xi^{+}$with a neighbourhood of all points on the imaginary axis below $\sigma=\alpha \pi i / 2$ excluded, and $\eta_{2}^{-}(u, \sigma)$ is bounded by (4.7) for $\sigma$ in $\Xi_{0} \cup \Xi^{-}$with a neighbourhood of all points on the imaginary axis above $\sigma=-\alpha \pi i / 2$ excluded.

From the bound (4.7), we infer that the error terms $\eta_{1}(u, \sigma), \eta_{2}^{+}(u, \sigma)$, and $\eta_{2}^{-}(u, \sigma)$ are $O\left(u^{-1}\right)$ as $u \rightarrow \infty$, and $O\left(\sigma^{-1}\right)$ as $\sigma \rightarrow \infty$, in their respective regions of asymptotic validity. The importance of the three $\mathrm{L}-\mathrm{G}$ solutions $L_{1}(u, \sigma), L_{2}^{+}(u, \sigma)$, and $L_{2}^{-}(u, \sigma)$ is that they are recessive in $\Xi_{0}, \Xi^{+}$, and $\Xi^{-}$, respectively.

We observe that although $L_{2}^{+}(u, \sigma)$ and $L_{2}^{-}(u, \sigma)$ have a similar (exponentially large) asymptotic form in $\Xi_{0}$, they are in general independent of one another. Note that the region of asymptotic validity of $L_{2}^{+}(u, \sigma)$ does not include any points in $\Xi^{-}$ (which contains the negative imaginary axis), and for points $\sigma$ close to the negative imaginary axis the bound is poor. A similar observation can be made regarding the region of asymptotic validity of $L_{2}^{-}(u, \sigma)$.

An important problem is to establish the linear relationship between the three functions, i.e., to determine constants $C$ and $D$ such that

$$
L_{2}^{+}(u, \sigma)=C L_{2}^{-}(u, \sigma)+D L_{1}(u, \sigma)
$$

The determination of $C$ can be achieved directly from the definitions of the functions and the error bounds by letting $\sigma \rightarrow \infty$ in $\Xi_{0}$ (so that $L_{1}(u, \sigma)$ vanishes). The determination of $D$ by a similar method would require letting $\sigma \rightarrow \infty$ so that either $L_{2}^{+}(u, \sigma)$ or $L_{2}^{-}(u, \sigma)$ vanishes $\left(\sigma \rightarrow \infty\right.$ in $\Xi^{+}$or $\left.\Xi^{-}\right)$, or at least so that all three functions are of the same order of magnitude when $\sigma$ or $u$ is large (i.e., on the positive or negative imaginary axis). This cannot be done as the common region of validity of the three functions does not include any points of $\Xi^{+}$or $\Xi^{-}$.

We solve connection problems of this kind by determining the relationship of the L-G solutions with the Whittaker function solutions. To do this we first note that

$$
\sigma=\int_{0}^{\xi}\left\{\frac{\tau-\alpha}{\tau}\right\}^{1 / 2} d \tau
$$

which comes from (3.11), (4.1), and the relation

$$
\int_{0}^{\alpha}\left\{\frac{\alpha-\tau}{\tau}\right\}^{1 / 2} d \tau=\int_{0}^{\tilde{a}}\left\{\frac{\tilde{a}-s}{s(1-s)}\right\}^{1 / 2} d s
$$

(which follows from the definition (3.8) of $\alpha$ ). Thus, from (4.9), we find that as $\xi \rightarrow \infty$ in $\hat{\mathbf{D}}$

$$
\sigma=\xi-\frac{1}{2} \alpha \ln (4 \xi)-\frac{1}{2} \alpha+\frac{1}{2} \alpha \ln (\alpha)+\frac{1}{2} \alpha \pi i+O\left(\xi^{-1}\right)
$$

The following three identifications come from the fact that the functions are solutions of the differential equation (3.16), each pair having the same recessive behaviour 
at infinity:

$$
\begin{gathered}
\hat{W}^{(1)}(u, \alpha, \xi)=e^{-\pi i / 4}\left(\frac{\xi}{\xi-\alpha}\right)^{1 / 4} L_{1}(u, \sigma), \\
\hat{W}^{(4)}(u, \alpha, \xi)=e^{(u \alpha-3 / 4) \pi i}\left(\frac{\xi}{\xi-\alpha}\right)^{1 / 4} L_{2}^{+}(u, \sigma), \\
\hat{W}^{(4)}\left(u, \alpha, \xi e^{-2 \pi i}\right)=-e^{\pi i / 4}\left(\frac{\xi}{\xi-\alpha}\right)^{1 / 4} L_{2}^{-}(u, \sigma)
\end{gathered}
$$

The constants of proportionality were found by comparing both sides at infinity and utilizing (4.11).

We finally turn our attention to a very important connection problem, the determination of constants $\lambda_{1}^{+}, \lambda_{1}^{-}$, and $\lambda_{2}$ such that

$$
\begin{aligned}
& \hat{W}^{(0)}(u, \alpha, \xi)=\left(\frac{\xi}{\xi-\alpha}\right)^{1 / 4}\left[\lambda_{1}^{+} L_{1}(u, \sigma)+\lambda_{2} L_{2}^{+}(u, \sigma)\right] \\
& \hat{W}^{(0)}(u, \alpha, \xi)=\left(\frac{\xi}{\xi-\alpha}\right)^{1 / 4}\left[\lambda_{1}^{-} L_{1}(u, \sigma)+\lambda_{2} L_{2}^{-}(u, \sigma)\right] .
\end{aligned}
$$

Clearly once these constants are determined, the coefficients of (4.8) immediately follow.

By letting $\xi \rightarrow \infty e^{\pi i}$ in (4.15) and (4.16), then with the aid of (3.23), (3.33), (4.5), (4.6), and (4.11) we find that

$$
\lambda_{2}=-e^{-\pi i / 4}\left\{1+\delta_{0}\right\}
$$

where

$$
\delta_{0}=\lim _{\xi \rightarrow \infty \exp (\pi i)}\left\{\frac{\hat{\varepsilon}^{(0)}(u, \alpha, \xi)}{\hat{\mathcal{U}}_{u \alpha / 2,1 / 4}^{(0)}(2 u \xi)}\right\} .
$$

The existence of this limit is guaranteed by the existence of $\lambda_{2}$. We next use [3, equation (4.80)] to obtain a bound for $\delta_{0}$. First, from [3, equation (4.58)] with $j=0$, $l=1, k=u \alpha / 2, m=1 / 4$, and $z=2 u \xi$, we have

$$
\frac{\hat{E}_{u \alpha / 2,1 / 4}^{(0)}(2 u \xi)^{-1} \hat{M}_{u \alpha / 2,1 / 4}^{(0,1)}(2 u \xi)}{\left|\hat{\mathcal{U}}_{u \alpha / 2,1 / 4}^{(0)}(2 u \xi)\right|}=\operatorname{cosec}\left\{\hat{\theta}_{u \alpha / 2,1 / 4}^{(0,1)}(2 u \xi)\right\}
$$

Now, from the definitions [3, equations (4.54), (4.55)], we note the following properties of the weight functions as $\xi \rightarrow \infty e^{\pi i}$

$$
\hat{E}_{u \alpha / 2,1 / 4}^{(j)}(2 u \xi)\left|\hat{\mathcal{U}}_{u \alpha / 2,1 / 4}^{(j)}(2 u \xi)\right| \sim \hat{e}_{u \alpha / 2,1 / 4}^{(j)} \quad(j=0,1) .
$$

Thus, on employing (4.19), (4.20), and [3, equation (4.61)], we obtain

$$
\lim _{\xi \rightarrow \infty \exp (\pi i)}\left\{\frac{\hat{E}_{u \alpha / 2,1 / 4}^{(0)}(2 u \xi)^{-1} \hat{M}_{u \alpha / 2,1 / 4}^{(0,1)}(2 u \xi)}{\left|\hat{\mathcal{U}}_{u \alpha / 2,1 / 4}^{(0)}(2 u \xi)\right|}\right\}=\frac{\left[\left\{\hat{e}_{u \alpha / 2,1 / 4}^{(0)}\right\}^{2}+\left\{\hat{e}_{u \alpha / 2,1 / 4}^{(1)}\right\}^{2}\right]^{1 / 2}}{\hat{e}_{u \alpha / 2,1 / 4}^{(0)}},
$$

which, in conjunction with [3, equation (4.80)], yields the desired bound

$$
\left|\delta_{0}\right| \leq e_{0}\left[\exp \left\{v_{\delta}(u, \alpha)(u \alpha+1)^{1 / 3} u^{-1+\delta}\right\}-1\right]
$$


Here $e_{0}$ is a computable (finite) constant defined by

$$
e_{0}=\sup _{0 \leq k<\infty}\left[1+\left\{\frac{\hat{e}_{k, 1 / 4}^{(1)}}{\hat{e}_{k, 1 / 4}^{(0)}}\right\}^{2}\right]^{1 / 2}
$$

and $v_{\delta}(u, \alpha)$ is defined by

$$
v_{\delta}(u, \alpha)=\frac{1}{2} u^{-\delta} \hat{\kappa}_{1 / 4} \mathcal{V}_{0, \infty \exp (\pi i)}(\hat{F}(u, \alpha, \xi)),
$$

where $\delta, \hat{\kappa}_{1 / 4}$, and $\hat{F}$ are given by [3, equations (4.71)-(4.73)]; note that for each fixed positive $\delta$ the parameter $v_{\delta}(u, \alpha)$ is $O(1)$ as $u \rightarrow \infty$. We remark that in [3] and here we have made a number of simplifications in arriving at (4.22), contenting ourselves with a simpler but not the sharpest possible bound.

Later on we will require that $\lambda_{2} \neq 0$. Hence from now on we assume that $u$ (i.e., q) is sufficiently large so that $\left|\delta_{0}\right|<1$.

Having found $\lambda_{2}$, we shall determine $\lambda_{1}^{+}$by letting $\xi \rightarrow \infty$ along the curve $G H$, since this curve is contained in the common region of asymptotic validity of the three asymptotic solutions in (4.15), and $L_{1}(u, \sigma)$ does not vanish as $\xi \rightarrow \infty$ along this curve. The curve $G H$ corresponds to the vertical line $\Re(\sigma)=\chi,(\Im(\sigma) \geq \alpha \pi / 2)$, where

$$
\begin{aligned}
\chi & =\int_{\alpha}^{\alpha+1 /(2 u)}\left\{\frac{\tau-\alpha}{\tau}\right\}^{1 / 2} d \tau \\
& =\left(\frac{1}{4 u^{2}}+\frac{\alpha}{2 u}\right)^{1 / 2}-\frac{\alpha}{2} \ln \left\{\frac{\alpha}{2}+\frac{1}{2 u}+\left(\frac{1}{4 u^{2}}+\frac{\alpha}{2 u}\right)^{1 / 2}\right\}+\frac{\alpha}{2} \ln \left\{\frac{\alpha}{2}\right\}
\end{aligned}
$$

Note that $\chi=O\left(u^{-3 / 2}\right)$ as $u \rightarrow \infty$ (except when $\alpha=0$ in which case $\chi=1 /(2 u)$ ).

Allowing $\sigma \rightarrow \chi+i \infty$ in (4.15), and using (3.23), (3.33), (4.5), (4.6), (4.11), and (4.17), we arrive then at the expression

$$
\lambda_{1}^{+}=\left(\frac{2 e}{u \alpha}\right)^{u \alpha} \frac{e^{-u \alpha \pi i / 2} \Gamma(u \alpha / 2+3 / 4)}{\Gamma(3 / 4-u \alpha / 2)}+\delta_{0}^{+},
$$

where

$$
\delta_{0}^{+}=\lim _{\sigma \rightarrow \chi+i \infty}\left\{e^{-u \sigma} \hat{\varepsilon}^{(0)}(u, \alpha, \xi)+\delta_{0} e^{-\pi i / 4} e^{-2 u \sigma}\right\} .
$$

The existence of this limit is guaranteed by the known existence of $\lambda_{1}^{+}$. To bound $\delta_{0}^{+}$ we note from (4.11) that

$$
\left|\xi^{-u \alpha / 2} e^{u \xi}\right| \rightarrow e^{u \chi}(4 e / \alpha)^{u \alpha / 2},
$$

as $\xi \rightarrow \infty$ on $G H(\sigma \rightarrow \chi+i \infty)$. Therefore using (3.27), (3.33), (4.20), and (4.28), and noting that the weight functions $\hat{E}_{u \alpha / 2,1 / 4}^{(j)}(2 u \xi)$ are equal to unity on $G H$, one can show from [3, equation (4.60)] and the triangle inequality that

$$
\lim _{\xi \rightarrow \infty \text { on } G H}\left\{\hat{M}_{u \alpha / 2,1 / 4}^{(0,1)}(2 u \xi)\right\} \leq e^{u \chi} M_{0}+e^{-u \chi}+e^{u \chi},
$$


where

$$
M_{0}=\sup _{0 \leq k<\infty}\left\{\frac{\Gamma(k+3 / 4)}{\Gamma(3 / 4-k)}\left(\frac{e}{k}\right)^{2 k}\right\}
$$

The existence of $M_{0}$ is readily established by use of Stirling's formula. Therefore, from (4.22), (4.27), (4.29), and [3, equation (4.80)], we have the desired bound

$$
\begin{aligned}
&\left|\delta_{0}^{+}\right| \leq\left(M_{0}+e^{-2 u \chi}+1\right)\left(\exp \left\{v_{\delta}^{+}(u, \alpha)(u \alpha+1)^{1 / 3} u^{-1+\delta}\right\}-1\right) \\
&+e_{0} e^{-2 u \chi}\left(\exp \left\{v_{\delta}(u, \alpha)(u \alpha+1)^{1 / 3} u^{-1+\delta}\right\}-1\right)
\end{aligned}
$$

where $v_{\delta}^{+}(u, \alpha)$ is defined by (4.24) but with the path of variation taken instead along $G H$. Again, note that $v_{\delta}^{+}(u, \alpha)$ is $O(1)$ as $u \rightarrow \infty$ for each fixed positive $\delta$.

Finally, to determine $\lambda_{1}^{-}$we let $\xi \rightarrow \infty$ on $G^{\prime} H^{\prime}(\sigma \rightarrow \chi-i \infty)$ to similarly obtain from the relation (4.16)

$$
\lambda_{1}^{-}=-i\left(\frac{2 e}{u \alpha}\right)^{u \alpha} \frac{e^{u \alpha \pi i / 2} \Gamma(u \alpha / 2+3 / 4)}{\Gamma(3 / 4-u \alpha / 2)}+\delta_{0}^{-},
$$

where $\delta_{0}^{-}$is the constant given by

$$
\delta_{0}^{-}=\lim _{\sigma \rightarrow \chi-i \infty}\left\{e^{-u \sigma} \hat{\varepsilon}^{(0)}(u, \alpha, \xi)+\delta_{0} e^{-\pi i / 4} e^{-2 u \sigma}\right\}
$$

and it too is bounded by (4.31).

\section{Identification of asymptotic solutions with Mathieu functions}

We are now in a position to obtain uniform asymptotic approximations of Mathieu functions for $q \rightarrow \infty$ with (1.2) holding, by identifying them with the asymptotic solutions of the previous two sections. All the results presented in this section are uniformly valid for this parameter range.

It is convenient to introduce the following function which we shall frequently employ

$$
\hat{\Phi}(\xi)=\xi^{-1 / 4}\left(\frac{\xi-\alpha}{t-\tilde{a}}\right)^{1 / 4}
$$

where the first factor on the right-hand side is defined such that $\arg \left(\xi^{-1 / 4}\right)=$ $-\frac{1}{4} \arg (\xi)$, and the second is defined to be positive on the real interval $-\infty<\xi<\xi_{1}$, and analytic in $\hat{\mathbf{D}}$ (except at $\xi=\xi_{1}$ ). Recall that $0 \leq \arg (\xi) \leq 2 \pi$ in $\hat{\mathbf{D}}$.

Our first identification is

$$
m_{v}^{(0)}(z, q)=\hat{c}_{v}^{(0)}(q) \hat{\Phi}(\xi) \hat{W}^{(0)}(u, \alpha, \xi)
$$

where $m_{v}^{(0)}(z, q)$ is the Mathieu function defined by $(2.9)$, and $\hat{W}^{(0)}(u, \alpha, \xi)$ is the asymptotic solution defined by (3.23) and (3.24). Here $\hat{c}_{v}^{(0)}(q)$ is a constant, and the relationship (5.2) follows from the fact that both functions are solutions of Mathieu's equation (1.1) which are recessive at $z=\pi / 2(\xi=0)$. To determine $\hat{c}_{v}^{(0)}(q)$ we compare both sides of the equation at $z=\pi / 2$. From (3.1) and (3.22) we perceive that

$$
\xi^{1 / 2}=(\tilde{a} / \alpha)^{1 / 2}(z-\pi / 2)+O\left\{(z-\pi / 2)^{2}\right\} \quad(z \rightarrow \pi / 2),
$$


and hence, from (2.11), (3.26), (5.1), and (5.2), we obtain the desired expression

$$
\hat{c}_{v}^{(0)}(q)=\left(\frac{\alpha}{8 \tilde{a} u^{3}}\right)^{1 / 4} \frac{\gamma(u \alpha / 2) \Gamma(3 / 2)}{\Gamma(u \alpha / 2+3 / 4)} .
$$

On gathering the above results together, we arrive at the following asymptotic approximation which holds uniformly for $\xi \in \hat{\mathbf{D}}_{0}$

$$
m_{v}^{(0)}(z, q)=\left(\frac{\alpha}{8 \tilde{a} u^{3}}\right)^{1 / 4} \hat{\Phi}(\xi)\left\{M_{u \alpha / 2,1 / 4}(2 u \xi)+\frac{\gamma(u \alpha / 2) \Gamma(3 / 2)}{\Gamma(u \alpha / 2+3 / 4)} \hat{\varepsilon}^{(0)}(u, \alpha, \xi)\right\} .
$$

The next identification is that of the solutions which are recessive at $z=\pi / 2+i \infty$ $\left(\xi=\infty e^{\pi i}\right)$, namely

$$
m_{v}^{(3)}(z, q)=\hat{c}_{v}^{(1)}(q) \hat{\Phi}(\xi) \hat{W}^{(1)}(u, \alpha, \xi)
$$

where $\hat{c}_{v}^{(1)}(q)$ is a constant of proportionality which can be determined by comparing both sides at infinity; from (2.21), (3.1), (3.19), (3.27), and (5.6), we obtain

$$
\hat{c}_{v}^{(1)}(q)=\frac{e^{-u \alpha^{*}} e^{-v \pi i / 2}}{u^{1 / 2} \pi^{1 / 2}} .
$$

Hence, on substituting (3.23) and (3.25) into the above results, we arrive at the following asymptotic approximation which is uniformly valid for $\xi \in \hat{\mathbf{D}}$

$$
m_{v}^{(3)}(z, q)=\frac{e^{-u \alpha^{*}} e^{-v \pi i / 2}}{u^{1 / 2} \pi^{1 / 2}} \hat{\Phi}(\xi)\left\{e^{-\pi i / 4} \gamma(u \alpha / 2) W_{-u \alpha / 2,1 / 4}\left(2 u \xi e^{-\pi i}\right)+\hat{\varepsilon}^{(1)}(u, \alpha, \xi)\right\}
$$

An L-G approximation for $m_{v}^{(3)}(z, q)$ follows immediately from (4.5), (4.12), (5.1), (5.6), and (5.7); the following approximation

$$
m_{v}^{(3)}(z, q)=\frac{e^{-u \alpha^{*}} e^{-(2 v+1) \pi i / 4}}{u^{1 / 2} \pi^{1 / 2}}(t-\tilde{a})^{-1 / 4} e^{u \sigma}\left\{1+\eta_{1}(u, \sigma)\right\}
$$

is uniformly valid for all $\sigma \in \Xi$ except for neighbourhoods of the points $\sigma= \pm \alpha \pi i / 2$.

Similar identifications can be made for the solutions that are recessive in the $\xi$ domains $\hat{\mathbf{D}}^{+}$and $\hat{\mathbf{D}}^{-}$. By proceeding in a similar manner to the derivation of (5.6) and (5.7), we obtain

$$
m_{v}^{(4)}(z, q)=\hat{c}_{v}^{(4)}(q) \hat{\Phi}(\xi) \hat{W}^{(4)}(u, \alpha, \xi)
$$

where

$$
\hat{c}_{v}^{(4)}(q)=-\frac{e^{u \alpha^{*}} e^{v \pi i / 2} e^{-u \alpha \pi i}}{u^{1 / 2} \pi^{1 / 2}}
$$

and

$$
m_{v}^{(3)}(z+\pi, q)=\hat{c}_{v}^{(4,-)}(q) \hat{\Phi}(\xi) \hat{W}^{(4)}\left(u, \alpha, \xi e^{-2 \pi i}\right)
$$

where

$$
\hat{c}_{v}^{(4,-)}(q)=-\frac{e^{u \alpha^{*}} e^{-v \pi i / 2}}{u^{1 / 2} \pi^{1 / 2}}
$$


Therefore, we deduce that the following approximation holds uniformly for $\xi \in \hat{\mathbf{D}}^{+}$

$$
m_{v}^{(4)}(z, q)=-\frac{e^{u \alpha^{*}} e^{v \pi i / 2} e^{-u \alpha \pi i}}{u^{1 / 2} \pi^{1 / 2}} \hat{\Phi}(\xi)\left\{\frac{e^{(u \alpha / 2-3 / 4) \pi i}}{\gamma(u \alpha / 2)} W_{u \alpha / 2,1 / 4}(2 u \xi)+\hat{\varepsilon}^{(4)}(u, \alpha, \xi)\right\}
$$

and the following holds uniformly for all $\sigma$ in $\Xi_{0} \cup \Xi^{+}$except for a neighbourhood of all points on the imaginary axis below (and including) $\sigma=\alpha \pi i / 2$

$$
m_{v}^{(4)}(z, q)=\frac{e^{u \alpha^{*}} e^{(2 v+1) \pi i / 4}}{u^{1 / 2} \pi^{1 / 2}}(t-\tilde{a})^{-1 / 4} e^{-u \sigma}\left\{1+\eta_{2}^{+}(u, \sigma\} .\right.
$$

Likewise, for $\xi \in \hat{\mathbf{D}}^{-}$we have arrived at the uniform approximation

$$
\begin{aligned}
m_{v}^{(3)}(z+\pi, q)=- & \frac{e^{u \alpha^{*}} e^{-v \pi i / 2}}{u^{1 / 2} \pi^{1 / 2}} \hat{\Phi}(\xi) \\
& \times\left\{\frac{e^{(u \alpha / 2-3 / 4) \pi i}}{\gamma(u \alpha / 2)} W_{u \alpha / 2,1 / 4}\left(2 u \xi e^{-2 \pi i}\right)+\hat{\varepsilon}^{(4)}\left(u, \alpha, \xi e^{-2 \pi i}\right)\right\}
\end{aligned}
$$

and for all $\sigma$ in $\Xi_{0} \cup \Xi^{-}$except for a neighbourhood of all points on the imaginary axis above (and including) $\sigma=-\alpha \pi i / 2$, we obtain

$$
m_{v}^{(3)}(z+\pi, q)=\frac{e^{u \alpha^{*}} e^{-(2 v-1) \pi i / 4}}{u^{1 / 2} \pi^{1 / 2}}(t-\tilde{a})^{-1 / 4} e^{-u \sigma}\left\{1+\eta_{2}^{-}(u, \sigma)\right\} .
$$

The completes the construction of uniform asymptotic approximations for the four recessive Mathieu functions. These allow us now to solve the connection problem (2.36), which we then use in conjunction with the other connection formulae to derive asymptotic approximations for the Floquet Mathieu function defined by (2.1). The second Floquet solution (2.5) can be similarly treated, as can other solutions of Mathieu's equation, but we do not pursue this.

The coefficients $A$ and $B$ of (2.36) are found immediately by comparing (4.12), (4.13), and (4.15) with (5.2), (5.6), and (5.10). As a result we deduce that

$$
m_{v}^{(0)}(z, q)=\frac{e^{\pi i / 4} \lambda_{1}^{+} \hat{c}_{v}^{(0)}(q)}{\hat{c}_{v}^{(1)}(q)} m_{v}^{(3)}(z, q)+\frac{e^{(3 / 4-u \alpha) \pi i} \lambda_{2} \hat{c}_{v}^{(0)}(q)}{\hat{c}_{v}^{(4)}(q)} m_{v}^{(4)}(z, q),
$$

and similarly, by comparing (4.12), (4.14), and (4.16) with (5.2), (5.6), and (5.12), we obtain

$$
m_{v}^{(0)}(z, q)=\frac{e^{\pi i / 4} \lambda_{1}^{-} \hat{c}_{v}^{(0)}(q)}{\hat{c}_{v}^{(1)}(q)} m_{v}^{(3)}(z, q)-\frac{e^{-\pi i / 4} \lambda_{2} \hat{c}_{v}^{(0)}(q)}{\hat{c}_{v}^{(4,-)}(q)} m_{v}^{(3)}(z+\pi, q) .
$$

We also can find the connection coefficient $k_{v}(q)$ of (2.29) by using (2.31) and setting $z=0$ and $z=\pi$ in the L-G approximation (5.9). Thus, on recalling that $z=0$ corresponds to $\sigma=\alpha^{*}-\alpha \pi i / 2$ and $t=1-i 0$, and $z=\pi$ corresponds to $t=1+i 0$ and $\alpha^{*}+\alpha \pi i / 2$, we arrive at the approximations

$$
m_{v}^{(3)}(\pi, q)=\frac{e^{-(2 v+1) \pi i / 4}}{u^{1 / 2} \pi^{1 / 2}}(1-\tilde{a})^{-1 / 4} e^{u \alpha \pi i / 2}\left\{1+\eta_{1}\left(u, \alpha^{*}+\alpha \pi i / 2\right)\right\}
$$


and

$$
e^{v \pi i} m_{v}^{(3)}(0, q)=\frac{e^{(2 v+1) \pi i / 4}}{u^{1 / 2} \pi^{1 / 2}}(1-\tilde{a})^{-1 / 4} e^{-u \alpha \pi i / 2}\left\{1+\eta_{1}\left(u, \alpha^{*}-\alpha \pi i / 2\right)\right\}
$$

Therefore, if we write

$$
1+\eta_{1}\left(u, \alpha^{*}+\alpha \pi i / 2\right)=\left(1+\delta_{1}\right) e^{i \pi \phi_{1}}
$$

with $\delta_{1}$ and $\phi_{1}$ real and $\phi_{1} \in(-\pi, \pi]$, we obtain the desired expression

$$
k_{v}(q)=\frac{1}{2} i e^{-v \pi i} u^{1 / 2} \pi^{1 / 2}(1-\tilde{a})^{1 / 4}\left(1+\delta_{1}\right)^{-1} \operatorname{cosec}\left\{\left(2 v-2 u \alpha-4 \phi_{1}+1\right) \pi / 4\right\}
$$

where $\delta_{1}$ and $\phi_{1}$ are $O\left(u^{-1}\right)$ as $u \rightarrow \infty$, and can be explicitly bounded by using (4.7) and (5.22).

We now are in a position to derive asymptotic approximations for the Floquet solution. We first note that the corresponding expression for $k_{-v}(q)$ is given by $(5.23)$ with $v$ replaced by $-v$. Consequently, from $(2.30),(5.6)$, and (5.10), we arrive at the approximation

$$
\begin{gathered}
m e_{v}(z, q)=\frac{i}{2} m e_{v}(0, q)\left(1+\delta_{1}\right)^{-1} \operatorname{cosec}\left\{\left(2 v+2 u \alpha+4 \phi_{1}-1\right) \pi / 4\right\}(1-\tilde{a})^{1 / 4} \hat{\Phi}(\xi) \\
\times\left[e^{u \alpha^{*}} e^{-(2 u \alpha-v) \pi i / 2} \hat{W}^{(4)}(u, \alpha, \xi)-e^{-u \alpha^{*}} e^{3 v \pi i / 2} \hat{W}^{(1)}(u, \alpha, \xi)\right]
\end{gathered}
$$

which is uniformly valid for $\xi \in \hat{\mathbf{D}}^{+}$, and so in particular can be used for real values of $z$ lying in the interval $\pi / 2+z^{*} \leq z \leq \pi$, where $z=\pi / 2+z^{*}$ corresponds to $\xi=\alpha+1 /(2 u)$.

Next we use (2.25) and (2.30) to obtain

$$
m e_{v}(z, q)=k_{-v}(q) m e_{v}(0, q)\left[m_{v}^{(3)}(z+\pi, q)-e^{-v \pi i} m_{v}^{(3)}(z, q)\right],
$$

which, with (5.6), (5.12), and (5.23) (with $v$ replaced by $-v$ ), yields the following asymptotic representation which is uniformly valid for $\xi \in \hat{\mathbf{D}}^{-}$

$$
\begin{aligned}
m e_{v}(z, q)=\frac{i}{2} & m e_{v}(0, q)\left(1+\delta_{1}\right)^{-1} \operatorname{cosec}\left\{\left(2 v+2 u \alpha+4 \phi_{1}-1\right) \pi / 4\right\}(1-\tilde{a})^{1 / 4} \hat{\Phi}(\xi) \\
& \times\left[e^{u \alpha^{*}} e^{v \pi i / 2} \hat{W}^{(4)}\left(u, \alpha, \xi e^{-2 \pi i}\right)+e^{-u \alpha^{*}} e^{-v \pi i / 2} \hat{W}^{(1)}(u, \alpha, \xi)\right]
\end{aligned}
$$

This expression can be used for real values of $z$ lying in the interval $0 \leq z \leq \pi / 2-z^{*}$.

Finally, from (2.30) and (5.18), we find that

$$
\begin{aligned}
m e_{v}(z, q)= & m e_{v}(0, q) k_{-v}(q)\left[\frac{e^{-(v-u \alpha+3 / 4) \pi i} \hat{c}_{v}^{(4)}(q)}{\lambda_{2} \hat{c}_{v}^{(0)}(q)} m_{v}^{(0)}(z, q)\right. \\
& \left.+\left(e^{v \pi i}-\frac{\lambda_{1}^{+} e^{-(v-u \alpha+1 / 2) \pi i} \hat{c}_{v}^{(4)}(q)}{\lambda_{2} \hat{c}_{v}^{(1)}(q)}\right) m_{v}^{(3)}(z, q)\right] .
\end{aligned}
$$


This can then be used with (5.2), (5.6), and (5.23) (with $v$ replaced by $-v$ ) to yield

$$
\begin{aligned}
m e_{v}(z, q)=- & \frac{i}{2\left(1+\delta_{1}\right) \lambda_{2}} m e_{v}(0, q) \operatorname{cosec}\left\{\left(2 v+2 u \alpha+4 \phi_{1}-1\right) \pi / 4\right\}(1-\tilde{a})^{1 / 4} \hat{\Phi}(\xi) \\
& \times\left[\left\{e^{-u \alpha^{*}} e^{3 v \pi i / 2} \lambda_{2}+e^{u \alpha^{*}} e^{(v-1) \pi i / 2} \lambda_{1}^{+}\right\} \hat{W}^{(1)}(u, \alpha, \xi)\right. \\
& \left.\quad+e^{u \alpha^{*}} e^{(2 v+1) \pi i / 4} \hat{W}^{(0)}(u, \alpha, \xi)\right], \quad \text { (5.28) }
\end{aligned}
$$

which holds uniformly for $\xi \in \hat{\mathbf{D}}_{0}$, and can be used for real values of $z$ lying in the interval $\pi / 2-z^{*} \leq z \leq \pi / 2+z^{*}$.

\section{The characteristic exponent $v$}

Our final task is to determine the relationship among the parameters $a, q$, and the characteristic exponent $v$. In [4], this problem was tackled by considering only two solutions of Mathieu's equation, viz.

$$
\begin{aligned}
w_{0}(z) & =\left(\frac{\xi-\alpha}{\xi(\tilde{a}-t)}\right)^{1 / 4} \hat{W}^{(0)}(u, \alpha, \xi) \\
& =(\tilde{a}-t)^{-1 / 4}\left[\lambda_{1}^{ \pm} L_{1}(u, \sigma)+\lambda_{2} L_{2}^{ \pm}(u, \sigma)\right], \\
w_{1}(z) & =(\tilde{a}-t)^{-1 / 4} L_{1}(u, \sigma) .
\end{aligned}
$$

The desired (asymptotic) relationship then was obtained by solving for $M^{ \pm 1}=e^{ \pm v \pi i}$ as the eigenvalues of the matrix

$$
\mathbf{A}=\left(\begin{array}{ll}
a_{11} & a_{12} \\
a_{21} & a_{22}
\end{array}\right)
$$

whose coefficients are given by the relations

$$
\begin{aligned}
& w_{0}(z+\pi)=a_{11} w_{0}(z)+a_{12} w_{1}(z) \\
& w_{1}(z+\pi)=a_{21} w_{0}(z)+a_{22} w_{1}(z)
\end{aligned}
$$

see $[1$, p. 30].

In this paper, we take a more direct approach by utilizing the connection formulae (5.18) and (5.19) that we have obtained. First, we eliminate $m_{v}^{(0)}(z, q)$ from these two relations to arrive at

$$
m_{v}^{(3)}(z+\pi, q)=\frac{e^{\pi i / 2} \hat{c}_{v}^{(4,-)}\left\{\lambda_{1}^{-}-\lambda_{1}^{+}\right\}}{\lambda_{2} \hat{c}_{v}^{(1)}(q)} m_{v}^{(3)}(z, q)+\frac{e^{-u \alpha \pi i} \hat{c}_{v}^{(4,-)}(q)}{\hat{c}_{v}^{(4)}(q)} m_{v}^{(4)}(z, q) .
$$

From (4.17), (4.26), (4.32), (5.7), (5.13), and the reflection formula for Gamma functions [10, Chapter 2, equation (1.07)], we obtain the following expression for the coefficient of $m_{v}^{(3)}(z, q)$ in (6.6)

$$
\frac{e^{\pi i / 2} \hat{c}_{v}^{(4,-)}\left\{\lambda_{1}^{-}-\lambda_{1}^{+}\right\}}{\lambda_{2} \hat{c}_{v}^{(1)}(q)}=e^{2 u \alpha^{*}}\left\{1+\delta_{0}\right\}^{-1}\left[G(u \alpha / 2) \cos (u \alpha \pi)+e^{3 \pi i / 4}\left(\delta_{0}^{-}-\delta_{0}^{+}\right)\right],
$$


where $G(k)$ is defined by

$$
G(k)=\frac{1}{\pi}\left(\frac{e}{k}\right)^{2 k} \Gamma(k+3 / 4) \Gamma(k+1 / 4) .
$$

This function is non-vanishing and bounded for $0 \leq k<\infty$, as is evident from Stirling's formula.

Next, from (5.11) and (5.13), we see that the coefficient of $m_{v}^{(4)}(z, q)$ in (6.6) is equivalent to

$$
\frac{e^{-u \alpha \pi i} \hat{c}_{v}^{(4,-)}(q)}{\hat{c}_{v}^{(4)}(q)}=e^{-v \pi i}
$$

On comparing the coefficients (6.7) and (6.9) of (6.6) with the corresponding ones of (2.25) we arrive at the following result:

Theorem 6.1. Define $\tilde{a}=\frac{1}{4}(a / q+2), u=\sqrt{q}$, parameters $\alpha$ and $\alpha^{*}$ by the elliptic integrals (3.9) and (3.21), respectively, and a bounded function $G(k)$ by (6.8). Then, for each $q>0$, the parameters $a, q$, and $v$ satisfy the relationship

$$
\cos (v \pi)=\frac{1}{2} e^{2 u \alpha^{*}}\left\{1+\delta_{0}\right\}^{-1}\left[G(u \alpha / 2) \cos (u \alpha \pi)+e^{3 \pi i / 4}\left\{\delta_{0}^{-}-\delta_{0}^{+}\right\}\right],
$$

uniformly for $-2 q \leq a \leq(2-d) q(d>0)$, where $\delta_{0}, \delta_{0}^{ \pm}$are bounded by (4.22) and (4.31), respectively; it is assumed that $q$ is sufficiently large so that $\left|\delta_{0}\right|<1$.

Since $\alpha=O(\tilde{a})$ as $\tilde{a} \rightarrow 0$, the bounds for $\delta_{0}, \delta_{0}^{ \pm}$show that these numbers are $O\left((u \tilde{a}+1)^{1 / 3} u^{-1+\delta}\right)$ uniformly for $0 \leq \tilde{a} \leq 1-d$ as $u \rightarrow \infty$, for arbitrary fixed positive $d$ and $\delta$.

In the special case when there exists a basically-periodic solution, that is, when $\cos (v \pi)= \pm 1$, we deduce from (6.10)

$$
\cos (u \alpha \pi)=\frac{1}{G(u \alpha / 2)}\left[e^{3 \pi i / 4}\left\{\hat{\delta}_{0}^{+}-\hat{\delta}_{0}^{-}\right\} \pm 2 e^{-2 u \alpha^{*}}\left\{1+\hat{\delta}_{0}\right\}\right] .
$$

In the present circumstances, $\alpha^{*}$ is positive and bounded away from zero. Therefore, the right-hand side of $(6.11)$ is $O\left((u \alpha+1)^{1 / 3} u^{-1+\delta}\right)$ as $u \rightarrow \infty$, and consequently

$$
\alpha=\frac{(2 m+1)}{2 u}+O\left((u \alpha+1)^{1 / 3} u^{-2+\delta}\right) \quad(m=0,1,2, \ldots),
$$

or equivalently

$$
\tilde{a} \int_{0}^{1}\left\{\frac{1-s}{s(1-\tilde{a} s)}\right\}^{1 / 2} d s=\frac{(2 m+1) \pi}{4 \sqrt{q}}+O\left((\sqrt{q} \tilde{a}+1)^{1 / 3} q^{-1+\delta}\right)
$$

uniformly for $0 \leq \tilde{a} \leq 1-d$ as $q \rightarrow \infty$. Equation (6.13) can be solved for $\tilde{a}$ from which the characteristic values of $a$ can be obtained by the relation $a=4 \tilde{a} q-2 q$. Explicit bounds are readily available for the order term in (6.13), and the approximation is valid for integer values $m$ satisfying

$$
0 \leq m \leq\{1-d\} \frac{2 \sqrt{q}}{\pi} \int_{0}^{1}\left\{\frac{1-s}{s(1-\{1-d\} s)}\right\}^{1 / 2} d s-\frac{1}{2} \quad(d>0) .
$$


Note that for the first few characteristic values $(m=O(1))$, the error term in (6.13) is $O\left(q^{-1+\delta}\right)$ as $q \rightarrow \infty$, whereas when $m=O\left(q^{1 / 2}\right)$ the error term in $(6.13)$ is $O\left(q^{-5 / 6+\delta}\right)$ as $q \rightarrow \infty$.

\section{References}

1. F. M. Arscott, Periodic Differential Equations, Pergamon Press, Oxford, 1964.

2. W. Barrett, Mathieu functions of general order: connection formulae, base functions and asymptotic formulae, (Parts I-V), Philos. Trans. Roy. Soc. London Ser. A, 30 (1981), 75-162.

3. T. M. Dunster, Uniform asymptotic solutions of second-order linear differential equations having a simple pole and a coalescing turning point in the complex plane, SIAM J. Math. Anal., to appear.

4. —_ Mathieu functions with large parameters: The eigenvalue problem, Proceedings of the International Symposium on Ordinary Differential Equations and Applications, Western Michigan University, 1993.

5. S. Goldstein, On the asymptotic expansion of the characteristic numbers of the Mathieu equation, Proc. Roy. Soc. Edinburgh Sect. A, 49 (1929), 210-223.

6. C. Hunter and B. Guerrieri, The eigenvalues of Mathieu's equation and their branch points, Stud. Appl. Math. 64 (1981), 113-141.

7. J. Meixner and F. W. Schäfke, Mathieusche Funktionen und Sphäroidfunktionen, Springer-Verlag, Berlin, 1954.

8. D. Naylor, On simplified asymptotic formulas for a class of Mathieu functions, SIAM J. Math. Anal. (6), 15 (1984), 1205-1213.

9. _— On a simplified asymptotic formula for the Mathieu function of the third kind, SIAM J. Math. Anal. (6), 18 (1987), 1616-1629.

10. F. W. J. Olver, Asymptotics and Special Functions, Academic Press, New York, 1974.

11. _ Second-order linear differential equations with two turning points, Philos. Trans. Roy. Soc. London Ser. A, 278 (1975), 137-174.

12. A. Sharples, Uniform asymptotic forms of modified Mathieu functions, Quart. J. Mech. Appl. Math. 20 (1967), 365-380.

Department of Mathematical Sciences, San Diego State University, San Diego, CaliforNIA 92182-0314, U.S.A. 